\title{
CLINICAL AUDIT: ADHERENCE TO EMPIRIC ANTIBIOTIC LOCAL PROTOCOL IN PERITONITIS IN A SURGICAL ICU. ARE WE IMPROVING?
}

Navarro-Echevarría P, Fernández-Téllez L, Rodríguez-Esteve A, Bernardino-Santos M, Olivera-Moreno D, García del Valle y Manzano S. University Hospital Fundación Alcorcón (Madrid-Spain). Department of Anaesthesiology and Intensive Care

\section{Background and Goal}

Adequate and early empiric antibiotic treatment and surgical source control improves the prognosis of patients with peritonitis. The aim of this second audit is to review the fulfilment of the Local Intraabdominal Infection protocol adapted to local flora approved in 2013 by the Infection and Antibiotic Policy Commission and to compare the results with a previous audit $(2015)^{1}$.

\section{Material and Methods}

We selected patients with peritonitis admitted at our surgical ICU (January 2015 to December 2016). Following data were collected: age, sex, isolated microorganism in cultures and antibiogram, temperature, hypotension, vasoactive treatment, tachycardia, tachypnea, leukocytosis or leukopenia, mechanical ventilation, renal substitutive treatment, lactate $>2 \mathrm{mmol} / \mathrm{L}$, high risk factors for worse clinical outcomes, empiric antibiotic treatment and who initiated it. We compared the empiric treatment administered with the local protocol recommendation and the present adherence to protocol with the previous audit.

\begin{tabular}{|c|c|c|c|c|c|}
\hline $\begin{array}{l}\text { Community acquired } \\
\text { intrabdominal infection }\end{array}$ & Mild-moderate & Severe & $\begin{array}{l}\text { Nosocomial } \\
\text { intrabdominal infection }\end{array}$ & Secondary PERITONITIS & Tertiary PERITONITIS \\
\hline \multicolumn{3}{|c|}{ Sampling in the operating room } & \multicolumn{3}{|c|}{ Sampling in the operating room } \\
\hline $\begin{array}{l}\text { No risks factors for poor } \\
\text { outcome }\end{array}$ & $\begin{array}{l}\text { - Amoxicillin-clavulanic } \\
\text { acid } 2 \mathrm{~g} / 8 \mathrm{~h}\end{array}$ & $\begin{array}{l}\text { Piperacillin- } \\
\text { Tazobactam 4-0,5/6-8h }\end{array}$ & \multirow[t]{2}{*}{$\begin{array}{l}\text { No risks factors for poor } \\
\text { outcome }\end{array}$} & $\begin{array}{l}\text { - Piperacillin- } \\
\text { Tazobactam 4-0,5/6- } \\
\text { 8h }\end{array}$ & \multirow{4}{*}{$\begin{array}{l}\text { - Imipenem } 1 \mathrm{~g} / 6-8 \mathrm{~h} \text { or } \\
\text { Meropenem } 1 \mathrm{~g} / 8 \mathrm{~h} \text { (if } \\
\text { Renal Failure) } \\
\text { + Vancomycin or } \\
\text { Linezolid } 600 \mathrm{mg} / 12 \mathrm{~h} \\
\text { + Fluconazole } 400 \text { - } \\
\text { 800mg/24h }\end{array}$} \\
\hline \multirow[t]{2}{*}{$\begin{array}{l}\text { With risks factors for } \\
\text { poor outcome }\end{array}$} & \multirow{2}{*}{$\begin{array}{l}\text { Ceftriaxone } 2 \mathrm{~g} / 24 \mathrm{~h} \\
+ \text { Metronidazole } \\
\text { So0mg/6h } \\
\text { - Ertapenem } 1 \mathrm{~g} / 24 \mathrm{~h} \text { (risk } \\
\text { of ESBL) }\end{array}$} & \multirow{2}{*}{$\begin{array}{l}\text { Imipenem } 1 \mathrm{~g} / 6-8 \mathrm{~h} \text { or } \\
\text { Meropenem } 2 \mathrm{~g} / 8 \mathrm{~h} \text { (if } \\
\text { Renal Failure) }\end{array}$} & & $\begin{array}{l}\text { +/-Fluconazole } 400 \text {. } \\
800 \mathrm{mg} / 24 \mathrm{~h}\end{array}$ & \\
\hline & & & \multirow[t]{2}{*}{$\begin{array}{l}\text { With risks factors for } \\
\text { poor outcome }\end{array}$} & $\begin{array}{l}\text { - Imipenem } 1 \mathrm{~g} / 6-8 \mathrm{gh} \text { or } \\
\text { Meropenem } 1 \mathrm{~g} / \mathrm{gh} \text { (if } \\
\text { Renal Failure) }\end{array}$ & \\
\hline \multirow{2}{*}{$\begin{array}{l}\text { Betalactam antibiotics } \\
\text { allergy }\end{array}$} & \multirow{2}{*}{$\begin{array}{l}\text { - Gentamicin } \\
5 \mathrm{mg} / \mathrm{kg} / 24 \mathrm{~h} \\
+ \text { Metronidazole } \\
500 \mathrm{mg} / 6 \mathrm{~h} \\
\text { - Tigecycline } 100 \mathrm{mg} \\
\text { firstly anf then } 50 \mathrm{mg} / 12 \mathrm{~h} \\
\text { (risk of ESBL.) }\end{array}$} & \multirow{2}{*}{$\begin{array}{l}\text { Tigecycline } 100 \mathrm{mg} \\
\text { firstly anf then } 50 \mathrm{mg} / 12 \mathrm{~h} \\
+ \text { Amikacin } \\
15 \mathrm{mg} / \mathrm{kg} / 24 \mathrm{~h}\end{array}$} & & $\begin{array}{l}\text { +/-Fluconazole } 400 \text { - } \\
\text { 800mg/24h }\end{array}$ & \\
\hline & & & $\begin{array}{l}\text { Betalactam antibiotic } \\
\text { allergy }\end{array}$ & $\begin{array}{l}\text { - Tigecycline } 100 \mathrm{mg} \\
\text { firsty anf then 50mg/12h } \\
+ \text { Amikacin } \\
15 \mathrm{mg} / \mathrm{kg} / 12 \mathrm{~h} \\
+1-\text { Fluconazole } 400- \\
800 \mathrm{mg} / 24 \mathrm{~h}\end{array}$ & $\begin{array}{l}\text { - Tigecycline 100mg } \\
\text { firstlyanf then 50mg/12h } \\
\text { + Amikacin } \\
15 \mathrm{mg} / \mathrm{kg} / 12 \mathrm{~h} \\
\text { + Fluconazole 400- } \\
800 \mathrm{mg} / 24 \mathrm{~h}\end{array}$ \\
\hline
\end{tabular}

\section{Results}

\begin{tabular}{|c|c|c|c|c|c|c|c|}
\hline & & \multicolumn{2}{|c|}{$\begin{array}{c}2013-2014 \\
n=48\end{array}$} & \multicolumn{2}{|c|}{$\begin{array}{c}2015-2016 \\
n=43\end{array}$} & \multirow[b]{2}{*}{8} & \multirow[b]{2}{*}{ p } \\
\hline & & $\mathrm{N}^{\circ}$ & $\%$ & $\mathrm{~N}^{0}$ & $\%$ & & \\
\hline \multirow{3}{*}{ High severity } & SIRS (22 criteria) & 41 & 85,42 & 34 & 79 & $\begin{array}{c}-0,064 \\
\text { Cl } 95 \%(-0,22 \text { to } 0,092)\end{array}$ & $>0,01$ \\
\hline & Need of vasoactive treatment & 35 & 72,92 & 18 & 41,86 & $\begin{array}{c}-0,301 \\
\text { Cl } 99 \%(-0,751 \text { to }-0,112)\end{array}$ & $<0,01$ \\
\hline & Lactate $>2$ & 36 & 75 & 24 & 55,8 & $\begin{array}{c}-0,192 \\
\text { Cl } 99 \%(-0,369 \text { to }-0,075)\end{array}$ & $<0,05$ \\
\hline \multicolumn{2}{|c|}{ Protocol fulfillment } & 25 & 52 & 20 & 46,5 & $\begin{array}{c}-0,05 \\
\text { Cl } 95 \%(-0,259 \text { to } 0,149)\end{array}$ & $>0,01$ \\
\hline \multicolumn{2}{|c|}{ Sample obtained during surgery } & 19 & 39,5 & 34 & 79 & $\begin{array}{c}0,395 \\
\mathrm{Cl} 99 \%(0,627 \text { to } 0,163)\end{array}$ & $<0,01$ \\
\hline
\end{tabular}

Start of empiric antibiotherapy

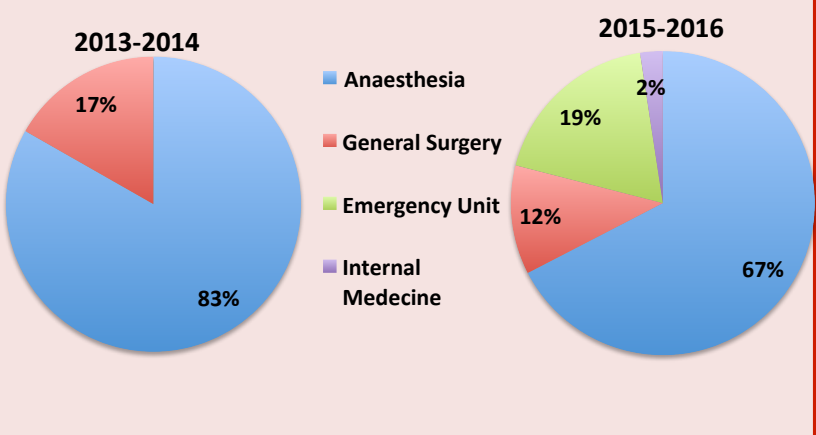

\section{Conclusions}

We achieved to double the percentage of intraoperative samples. This allows the later correct adjustment of the antibiotic treatment. Emergency and Surgery Units are much more involved and the first dose of antibiotic is given earlier. Actions to improve the fulfilment of the intraabdominal infection protocol must be taken in order to improve the adherence to local guideline. Finally, our protocol should be reviewed to adjust it to the new SOFA criteria².

Adherence to empiric antibiotic treatment in peritnotis in a surgical ICU. Olivera D. The European Anaesthesiology Congress.Berlin 2015

Rhodes A, Evans LE, Alhazzani W, et al. Surviving Sepsis Campaign: International Guidelines for Management of Sepsis and Septic Shock: 2016 Intensive Care Med 2017 